This item was submitted to Loughborough's Research Repository by the author.

Items in Figshare are protected by copyright, with all rights reserved, unless otherwise indicated.

\title{
Assessment of the impact sound in golf putting
}

PLEASE CITE THE PUBLISHED VERSION

http://dx.doi.org/10.1080/17461390500188728

\section{PUBLISHER}

(C) Taylor \& Francis Ltd.

\section{VERSION}

AM (Accepted Manuscript)

LICENCE

CC BY-NC-ND 4.0

\section{REPOSITORY RECORD}

Barrass, David F., Jonathan R. Roberts, and Roy Jones. 2019. "Assessment of the Impact Sound in Golf Putting". figshare. https://hdl.handle.net/2134/11477. 
This item was submitted to Loughborough's Institutional Repository (https://dspace.lboro.ac.uk/) by the author and is made available under the following Creative Commons Licence conditions.

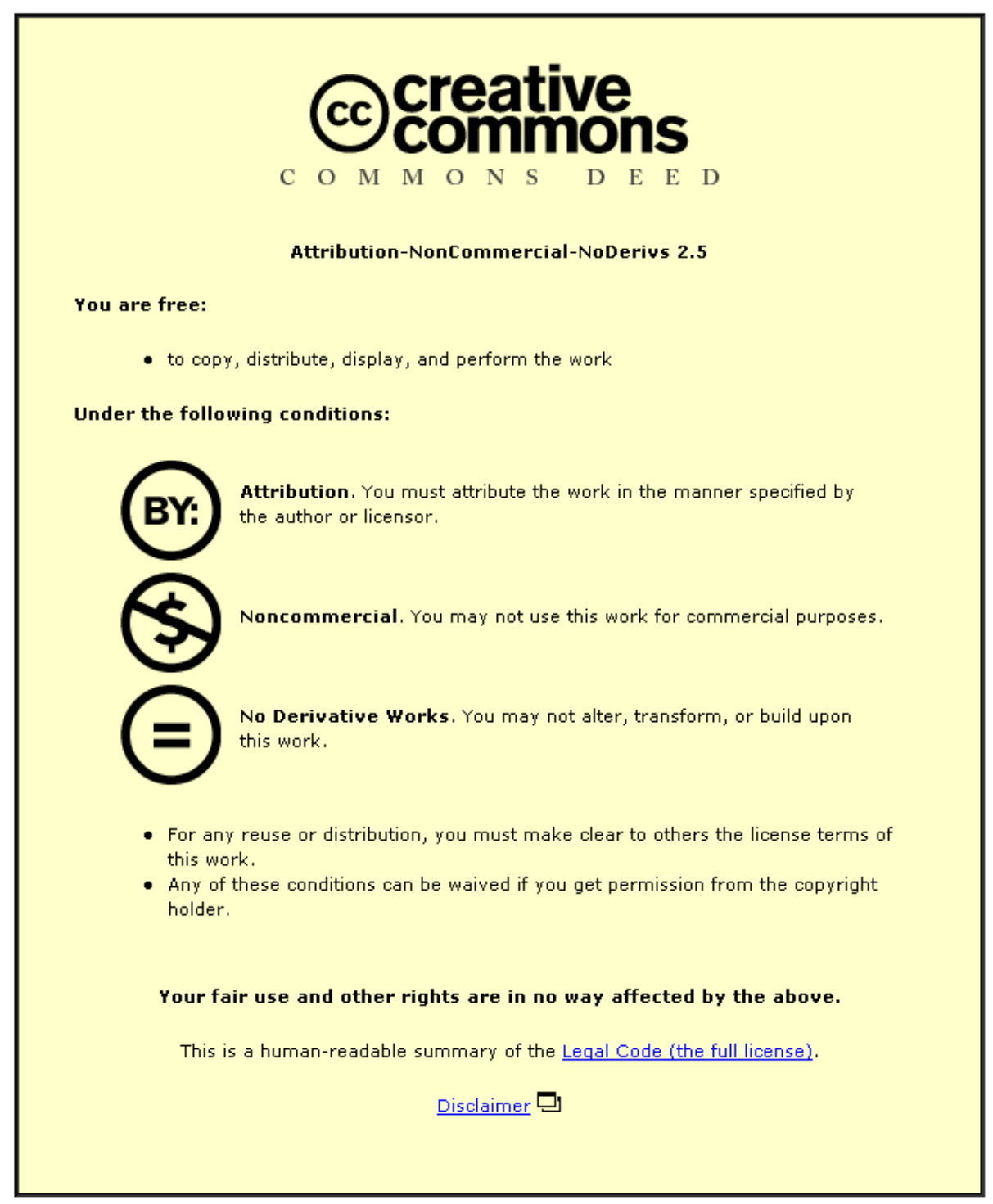

For the full text of this licence, please go to: http://creativecommons.org/licenses/by-nc-nd/2.5/ 


\title{
Assessment of the Impact Sound in Putting
}

\author{
D.F. BARRASS, J.R. ROBERTS, R. JONES \\ Wolfson School of Mechanical and Manufacturing Engineering, Loughborough University, Loughborough, \\ Leicestershire, LEn 3TU UK \\ KEYWORDS: Golf, putting, impact sound, perception
}

\begin{abstract}
The influence of impact sound in putting on players' perceptions of 'feel' is explored in this paper. Tests were conducted to investigate the impact sound characteristics of five different ball types using two different putter types. The first test studied the impact sound of purely the ball, while the second test investigated the influence of putter construction and impact location on impact sound for the different ball types. Trends were found between sound spectra peaks in the $2-4 \mathrm{kHz}$ range and the compression values of the balls; whilst frequency content was more dependent on putter type and impact location than on ball construction in the $0-2 \mathrm{kHz}$ range. The final test employed a paired comparison technique to investigate players' perceptions of sharpness and loudness of impact sound, ball speed from the clubface and ball hardness. Relationships between the subjective data and the sound characteristics of the balls were then examined. It was found that the ball the players' perceived to have the sharpest and loudest sound, feel the hardest and come off the clubface the quickest also had the largest calculated values of loudness and sharpness and had a spectral peak at a higher frequency than the other balls.
\end{abstract}

\section{Introduction}

Putting is a vital part of the game of golf, as it is usually the 'terminal' shot on every hole; a study by Pelz (1989) showed that, for players of handicaps up to 28 , putting typically accounts for $43 \%$ of shots played in a game. Despite its relative importance, however, few studies have investigated the role of the putter to the same degree as other clubs and scientific understanding is less well developed. Perhaps, because the putter does not apply as great a force during impact as other clubs, such as drivers and long irons, investigation into the mechanics of impact have typically been ignored. However, as the putter is considered a 'feel' club, it is important that players receive good feedback from the putter so they know how well their putt has been executed.

Hocknell et al. (1996) defined 'feel' as encompassing 'the physical and psychological feedback experienced by a golfer in playing a golf shot.' There are several different forms of feedback that will be received by the golfer including kinaesthetic feedback, which describes the positions and movements of their limbs, as well as tactile, auditory and visual sensations. Based on this the golfer will judge the quality of the shot and the characteristics of the equipment used.

A previous study investigated golfers' perceptions of feel during short game play by interviewing elite players whilst they were performing a series of pitching, chipping and putting exercises with a range of different balls. During the interviews, the golfers typically described both the feel of ball hardness and the speed at which the ball left the clubface. 'That felt a little softer for putting. [It] didn't seem quite as lively off the clubface. I think that came off the face slower.' These two characteristics appeared to be related and were often linked to ball control. In general, golfers perceived a softer ball to leave the clubface slower and as a result, they felt more in control of a shot than with a hard ball that they perceived to 'shoot' off the clubface. The ideal feel of ball hardness differed between the golfers, but for the majority it was towards the softer end of the scale.

'That one to me again had a slightly harder feel to it... I could feel it running away from me. I felt as though I wasn't in $100 \%$ control of the shot when it bounced and when I hit it up. The feeling I had off the club was as though it was bouncing off rather than gripping the club... sometimes it felt as if it was just going boing, jumping off the club a little bit.'

'The softer the ball is, the easier it is to judge the distance, I think. If it's hard, it seems to ping off the clubface.'

To achieve optimum performance when putting, therefore, it is essential that players are comfortable both physically and psychologically with their putter and ball. Designing equipment that golfers will be confident using requires an understanding of what occurs at impact and how these events influence the golfer and this knowledge needs to be developed.

'The trajectory of the ball in flight, the sensation in the hands and, most importantly the sound of the impact are the three main components of 'feel", according to Hocknell et al. (1996). For shots with a driver, J.R. 
Roberts et al. (submitted) have found relationships between the feel of a golf shot and the sound and vibration measured from impact. The sensations in the hands from putting impacts are considerably smaller than during full shots and it is anticipated, therefore, that the impact sound will be the predominant contributor to the feel of short game shots. Indeed, several of the golfers interviewed during the short game study related the perceived hardness of the ball to the impact sound.

'Straight away, the noise is different. It's a slightly higher pitched noise. It's more of a click than a thud, I guess, not a technical term. So my brain's telling me that it's a firmer ball and to not hit it quite as hard... [it's] definitely towards the firmer end of the scale.'

Axe et al. (2002) also described how golfers use auditory signals to determine the perceived qualities of a golf ball.

The sound of the impact of a golf ball is distinctive and knowledgeable players and spectators are able to distinguish the quality of a golf shot by the characteristic sound of the 'click'. Golfers who are curious about the properties of an unknown ball will often drop it onto a hard surface to 'hear' as well as see how it rebounds. (Axe et al., 2002, pp. 359)

The two papers by Axe et al. (2002) and Shannon and Axe (2002) looked at the vibration mode structure of a golf ball and the acoustic signature produced by a range of balls. The authors used numerical methods to estimate the natural frequencies of each mode shape and compared the results with the acoustic measurements when the balls were dropped onto a concrete surface. From the measured data, it was found that the first acoustic mode contributed most to the impact sound and had a natural frequency between 2.5 and $4.5 \mathrm{kHz}$ depending on ball type. The authors also discovered that the frequencies of the second and third modes could be predicted from the first as they were 1.53 and 2.08 times respectively, the frequency of the first mode.

The purpose of this study was to investigate the sound characteristics of both the ball and club during the impact of a putting stroke and their subsequent influence on player's perceptions of the equipment. Other prior studies have investigated the sound produced by golf impacts (Hocknell et al., 1996; J.R. Roberts et al., submitted) and human perceptions of a golf shot (Roberts, 2002). However, neither of these studies examined the influence of the putter. By analysing aspects of the ball and putter that contribute to the impact sound, knowledge can be developed of how these characteristics relate to a player's perception of 'feel' and how they can be engineered to induce satisfaction.

In this study, five different types of golf ball were investigated. The first stage of testing used a similar set up to a Newton's cradle, housed in an acoustic chamber, to investigate the sound characteristics of the balls alone. A second stage of testing investigated the interaction of the balls with two different types of putter using a pendulum mechanism to generate impact sounds. The final stage investigated players' perceptions of the different balls when putting so that the measured sound characteristics could be correlated with their responses.

\section{Measurement of ball sound characteristics}

\section{Ball Selection}

Five ball types were selected that were considered to represent a range of constructions and feel characteristics without possessing extreme properties. The properties of the balls that were selected are summarized in Table 1. From a twelve ball sample, five balls considered to be representative of each ball type were then selected based on their compression values. An industry standard compression test was adopted where a Lloyd Instruments Automated Materials Testing System applied an initial preload of $35.6 \mathrm{~N}(8 \mathrm{lbf})$ to the ball, followed by a further load of $409.2 \mathrm{~N}$ ( $92 \mathrm{lbf}$ ). The deflection of the ball, $\chi$, from the preload to the final load was measured in thousandths of an inch and the compression value then calculated using Equation (1).

$$
\text { Compression }=188-2 x
$$

Twelve balls of each type were loaded in three mutually perpendicular directions, using the manufacturer's logo to locate the first axis, and the deflections measured. A compression value was obtained from the mean deflection for each ball; the mean compression values for the five ball types are shown in Table 1. The five balls with compression values closest to the mean for that ball type were selected for use in the tests. The compression testing was conducted following guidelines from a major ball manufacturer, since there is no current international standard for ball compression measurement.

Table 1. Properties of the test balls.

\begin{tabular}{|c|c|c|c|c|}
\hline Ball & Cover & Construction & $\begin{array}{c}\text { Mass } \\
(\mathrm{g})\end{array}$ & $\begin{array}{c}\text { Mean measured } \\
\text { compression }\end{array}$ \\
\hline A & Surlyn & 2-piece & 45.8 & 75.1 \\
\hline B & Urethane & 3-piece & 45.9 & 92.0 \\
\hline C & Urethane & 3-piece & 45.8 & 70.7 \\
\hline D & Surlyn & 2-piece & 45.4 & 47.7 \\
\hline E & Surlyn & 2-piece & 45.6 & 61.3 \\
\hline
\end{tabular}

\section{Newton's Cradle Test}

When two objects collide the resultant sound is a function of the natural frequencies of the individual objects. To isolate the impact sound of a golf ball from the effects of another surface, sounds were generated by impacting one ball against another ball of the same type. The configuration used is similar to a two ball Newton's cradle, as illustrated in Figure 1. Each ball was suspended on two $0.15 \mathrm{~m}$ lengths of thread, attached to the top of the ball at the same point using a cyanoacrylite adhesive. The 
thread contact point was as small as practically possible to have minimal effect on the sound characteristics.

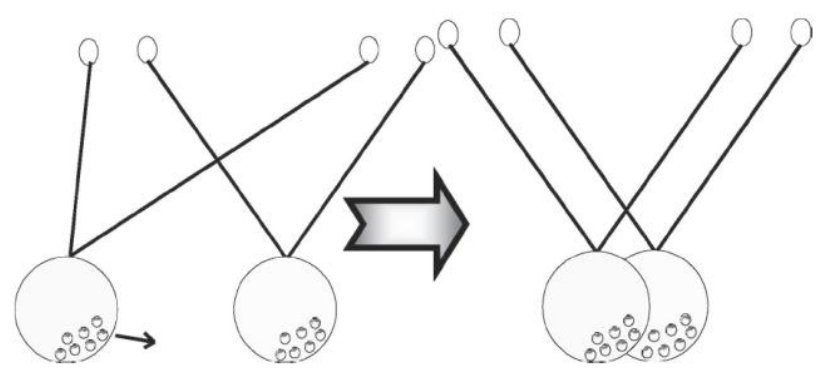

Figure 1. Diagram of Newton's cradle set-up for the balls.

The tests were conducted in a cabinet lined with acoustic foam tiles of thickness between 70 and $110 \mathrm{~mm}$, to reduce the effects of sound reflections, room acoustics and background noise. The cabinet set up is pictured in Figure 2.

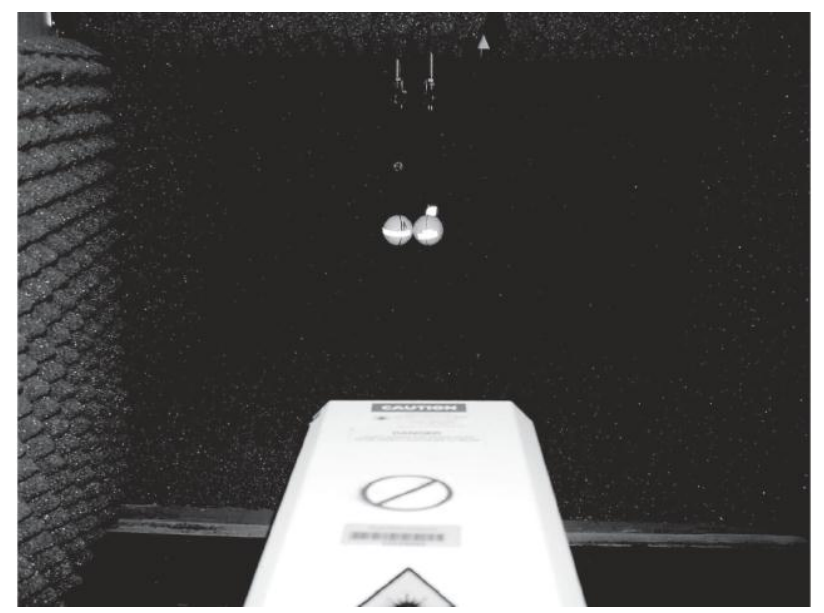

Figure 2. Balls suspended in an acoustic foam-lined cabinet.
A length of thread, attached to the opposite side of the ball to the impact area, was used to hold and release the first ball from a consistent position (an angle of $51^{\circ}$ from the vertical) each time. Fifteen impacts were conducted for each ball type; five impacts with each combination of three balls, selected from the initial five balls.

A microphone (Brüel and Kjær type 4155) was positioned 6omm from the point of collision to record the impact sound. In addition, a laser vibrometer was aligned to measure the vibration of the surface of the released ball, perpendicular to the direction of motion after impact. The laser was directed at the ball being released as, theoretically, this would be stationary after impact. Reflective tape was adhered to the ball to enhance the reflected laser energy.

Example frequency spectra of the sound and vibration from impact are illustrated in Figure 3. It can be seen that there are corresponding peaks in both spectra, confirming that the major components of the impact sound are produced by the vibrating surface of the ball.

The mean sound spectra for the balls are compared in Figure 4. In the $0-2 \mathrm{kHz}$ range there is very little difference between the sound pressure levels produced by the balls. From approximately $2.75 \mathrm{kHz}$ to $4 \mathrm{kHz}$; each ball type produces a peak at a different frequency; ball $\mathrm{D}$ has the lowest frequency peak, followed by ball $\mathrm{E}$, ball $\mathrm{A}$ and then ball $\mathrm{C}$ with ball $\mathrm{B}$ having the highest frequency at around $4 \mathrm{kHz}$. The peak order shows a relationship with the order of ball compression data in Table 1.

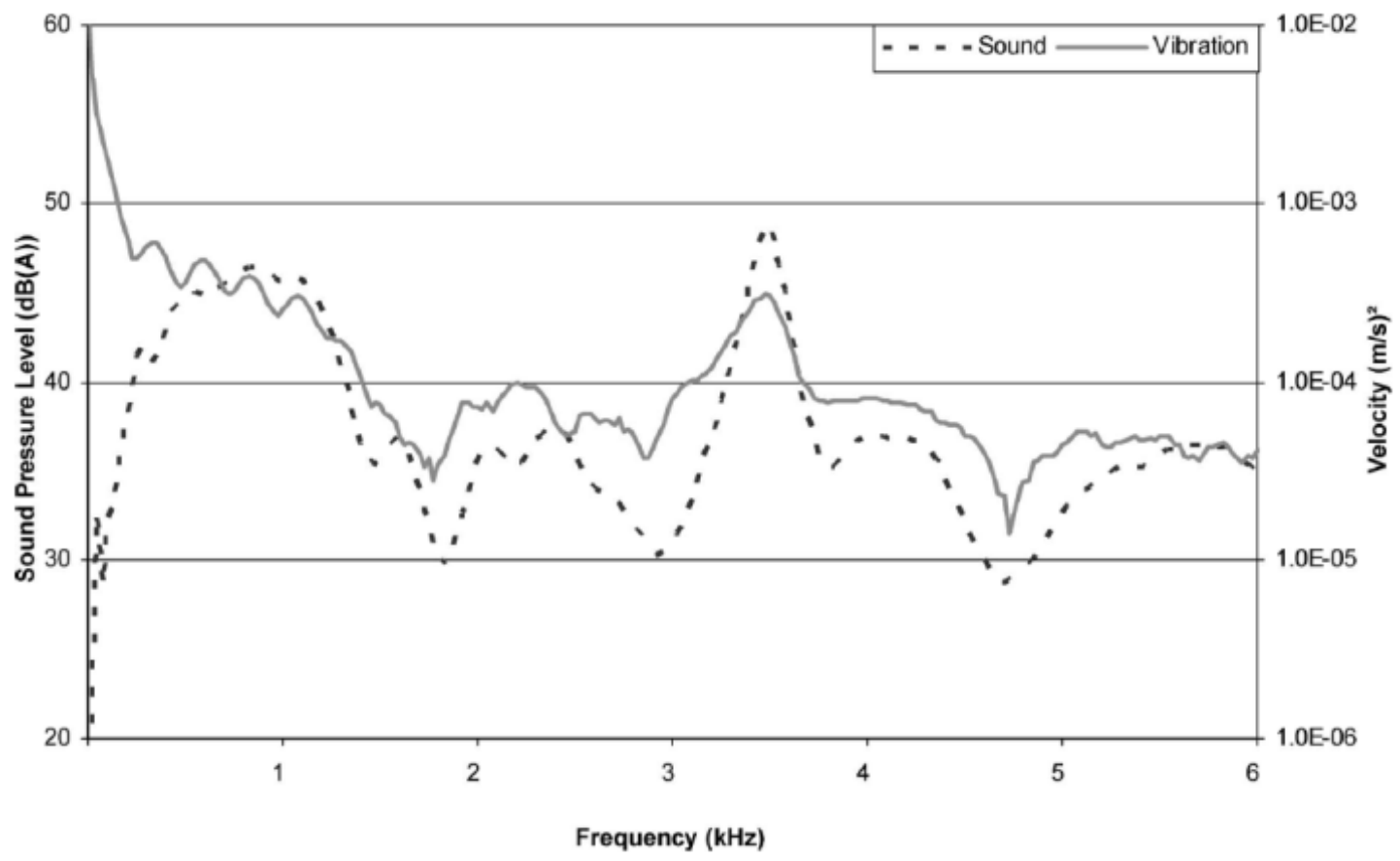

Figure 3. Example frequency spectra of sound and vibration data from an impact with ball type A. 


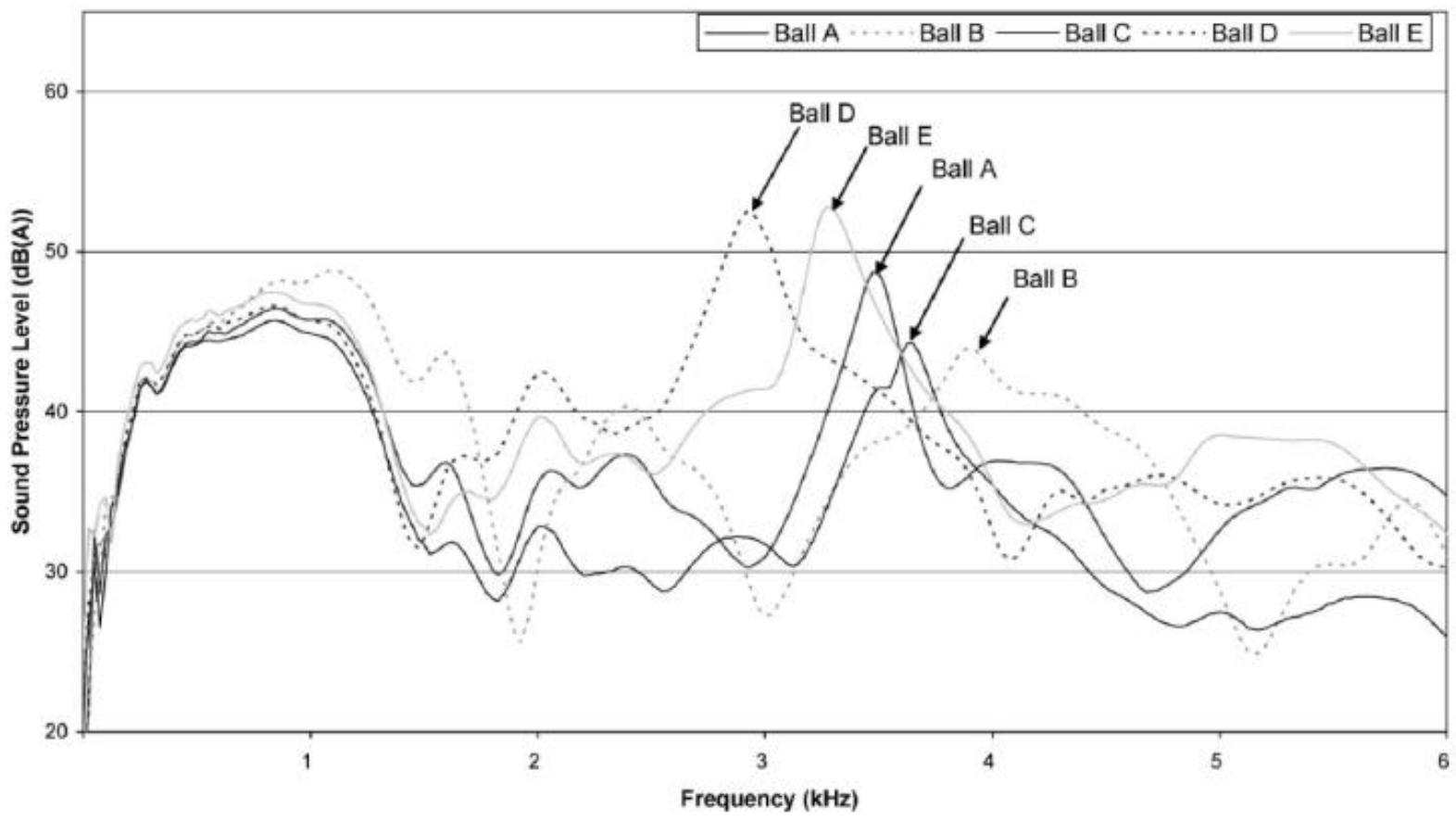

Figure 4. Comparison of mean sound spectra for five different ball types obtained from ball-on-ball impacts.

This frequency range also corresponds with the dominant sound frequencies measured by Shannon and Axe (2002) from ball drop tests, which they attributed to the first mode of ball vibration. There is, however, less evidence of second and third modes at 1.5 and 2 times the frequency of the first as suggested by Shannon and Axe (2002).

\section{Effect of putter head type and impact location on sound characteristics}

After establishing the sound characteristics of the different ball types, impact sounds were analysed during collisions with different putterheads. These measurements were then correlated with the perceptions of the golfers using the equipment.

The putter heads selected were:

1. A modern, face-balanced, polymer insert putter

2. A modern, face-balanced, steel putter

The testing was conducted indoors in a large room, in order to provide a consistent, quiet environment and to ensure that effects such as wind noise or reflected sound did not affect the quality of the sound recording. Preliminary tests with competent golfers confirmed that the room acoustics did not have a noticeable effect on the impact sound.

The putting pendulum device illustrated in Figure 5 was used to provide a consistent swing pattern and repeatable impact location. The release point of the pendulum arm required to produce a $6 \mathrm{~m}$ putt on the carpet surface was identified and used throughout the tests. This point corresponded to a clubhead speed prior to impact of approximately $0.8 \mathrm{~ms}^{-1}$. Measurements were taken for three impact locations - centre, heel and toe. The offcentre impacts were located $32 \mathrm{~mm}$ either side of the geometric centre of the putter face as illustrated in Figure 6; this represented the maximum distance towards the toe and heel that could be achieved with the ball striking the face insert on one of the putters. Five balls of each type were each struck three times at the three impact locations.

The A-weighted impact sound was measured using a Brüel \& Kjær Type 2231 sound level meter with a type 4155 microphone positioned close to the point of impact. A Data Physics two channel PCMCIA card was used to acquire $80 \mathrm{~ms}$ of data at a sample rate of $51.2 \mathrm{kHz}$, and a $20 \mathrm{kHz}$ low pass filter was used to prevent aliasing.

While little difference between balls can be seen in the o-2 $\mathrm{kHz}$ range of impact sound spectra from central impacts, considerable differences exist between each putter type, see Figure 7. The impact sound of the insert putter has greater frequency content in this range than the standard putter. In the $2-6 \mathrm{kHz}$ range, a similar trend can be seen for both putters. The peaks in the spectra are in the same order and are also at similar frequencies to the measurements from the Newton's cradle test. It can also be observed that the trend repeats itself at frequencies above $4 \mathrm{kHz}$. 


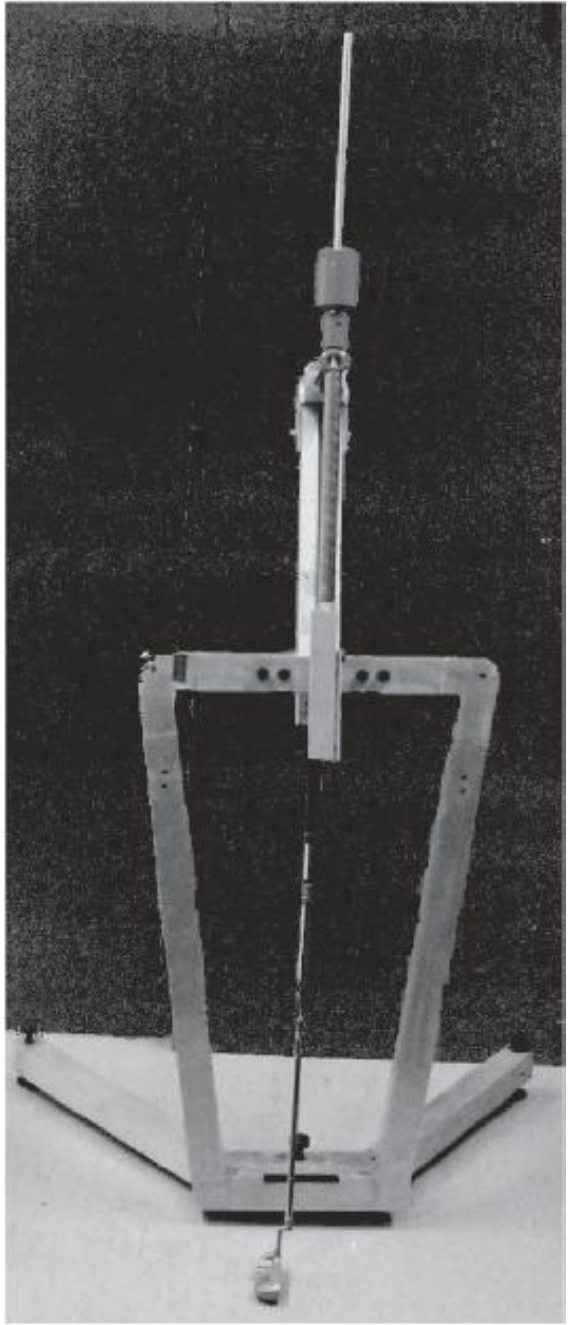

Figure 5. Putting pendulum mechanism.

Several trends are observed when analysing the mean sound pressure levels of different combinations of ball type, putter type and impact location, as can be seen in Figure 8. Regardless of impact location or putter type, ball $B$ produced the highest sound pressure levels (SPL). There are less significant differences in SPL between the other balls irrespective of the putter used or the impact location, though ball D generally produced the lowest SPL. It can also be seen that, for both the steel and insert putter; centre impacts produced the lowest SPLs followed by toe impacts, whilst the SPL of heel impacts was always significantly greater than the other two impact locations. The difference in SPL between the two putters is very small for centre and toe impacts, but the SPL of the heel impacts with the insert putter are significantly greater than with the steel putter, regardless of ball type.

A-weighted SPL is generally considered to estimate the perceived loudness of a sound, however, it has a number of limitations. It is based on subjective responses to low level pure tones and does not take into account the interaction between different components of a complex sound (Zwicker and Fastl, 1999). As a result, a method of

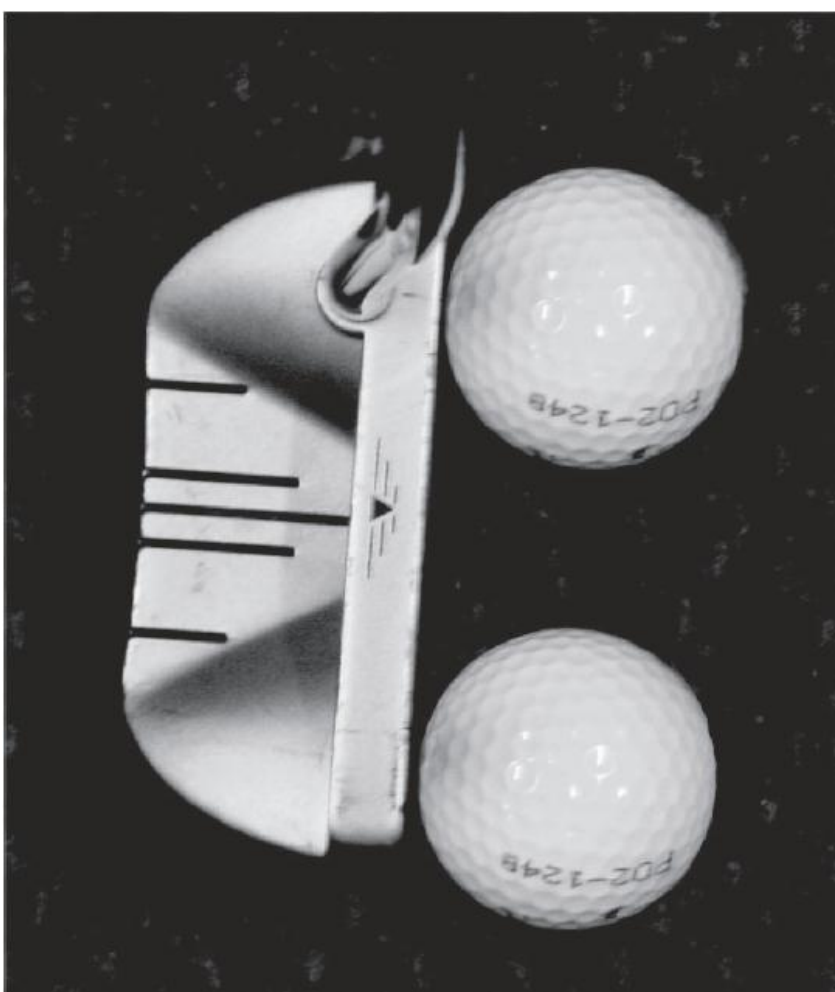

Figure 6. The two off-centre impact locations for the tests.

converting a sound spectrum into a specific loudness distribution has been developed, from which perceived sound qualities can be obtained. The area under the specific loudness distribution can be used as a measure of loudness and the weighted first moment of the spectrum describes the sharpness of the sound (ISO 532:1975; Zwicker and Fastl, 1999).

These two metrics were calculated for the centre impact sounds and the mean values for each ball type are shown in Figure 9. It can be seen that ball B has a significantly louder and sharper sound than balls C and D.

Figure 10 shows the influence of impact location on the mean sound spectra for each of the putters with ball B and illustrates typical trends that can be seen with all of the balls. In the o-2 $\mathrm{kHz}$ range, for the steel putter, peaks occurring at approximately $0.5 \mathrm{kHz}, 1.2 \mathrm{kHz}$ and $1.6 \mathrm{kHz}$ are mostly influenced by impact location, regardless of ball type. Similar trends follow for the insert putter peaks at $1.5 \mathrm{kHz}$ and $2 \mathrm{kHz}$. For both putters the magnitude of the peaks is greater with the off-centre impacts than with the centre impacts and with the insert putter it is particularly noticeable that the peaks in the range o-1.5 $\mathrm{kHz}$ are of greater magnitude with the heel impacts. Impact location appears to have less influence at higher frequencies, although heel impacts with the steel putter excite the higher frequencies more than centre and toe impacts. 

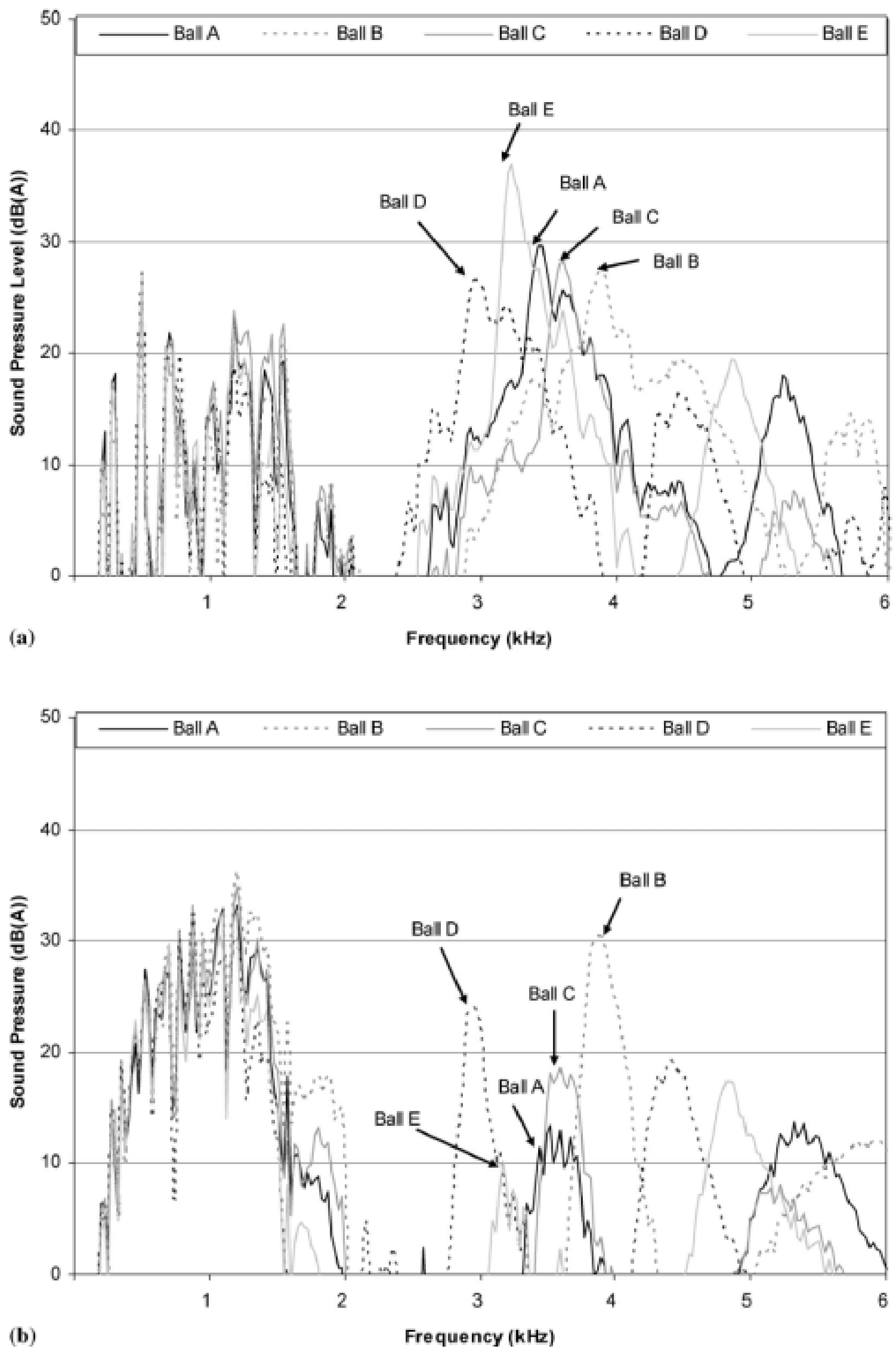

Figure 7. Mean spectra from central impacts with each ball type using two different putters: (a) steel putter, (b) insert putter. 


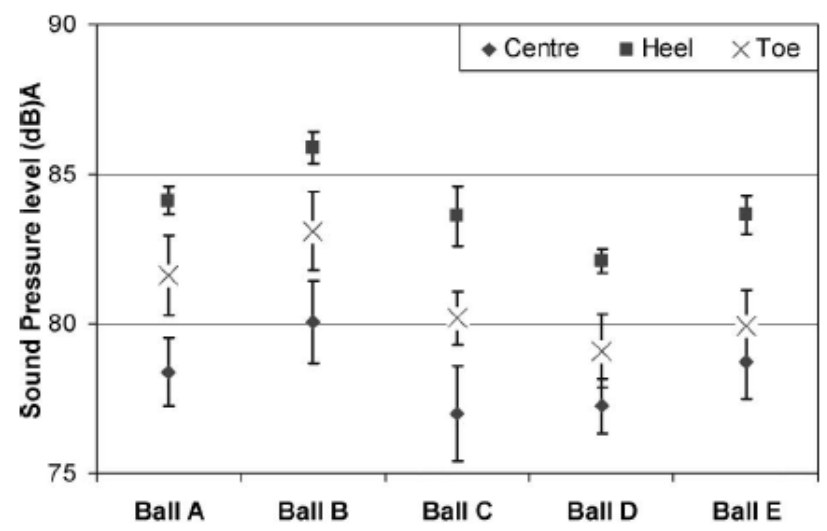

(a)

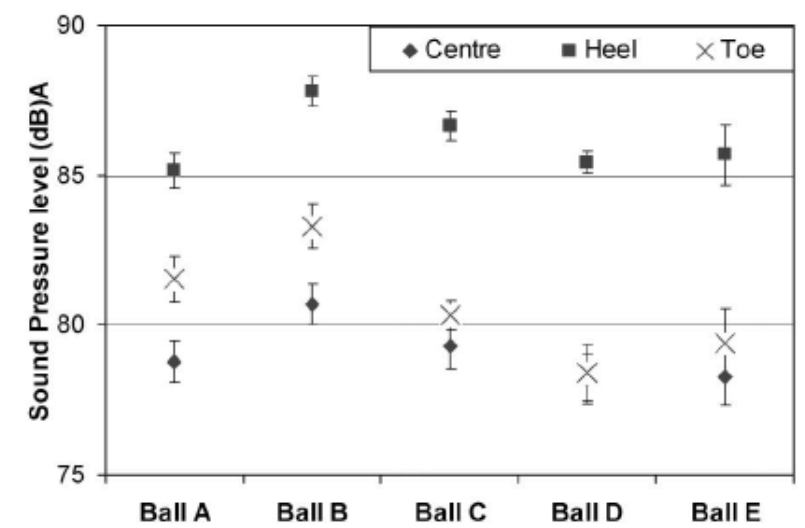

(b)

Figure 8. Mean sound pressures ( \pm 1 standard deviation) produced by five different ball types from three impact locations with two types of putter: (a) steel putter, (b) insert putter.

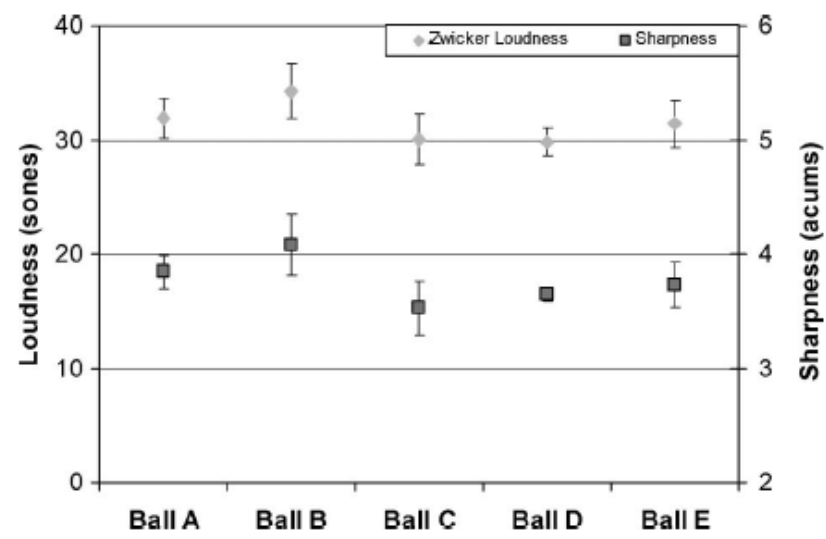

Figure 9. Mean Zwicker loudness and sharpness values $( \pm 1$ standard deviation) produced by central impacts with the steel putter.
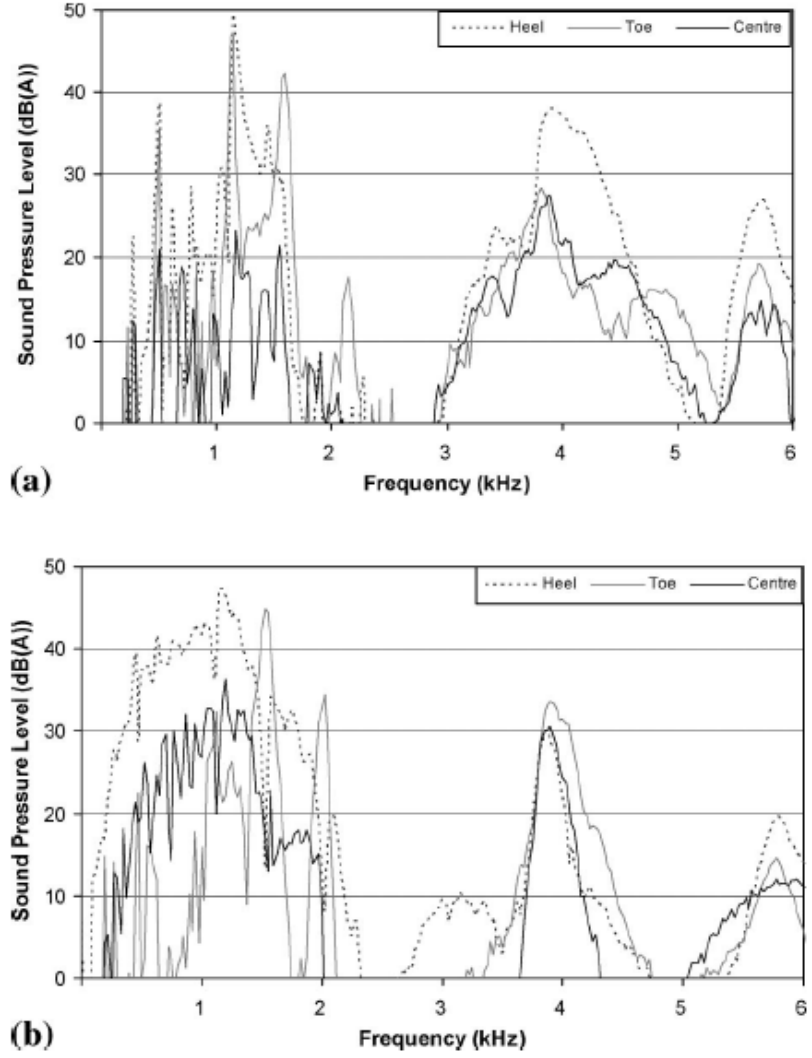

Figure 10. Mean spectra for impacts at three locations using ball B and two different types of putter: (a) steel putter, (b) insert putter.

\section{Effect of ball composition on players' perceptions}

The final stage in the study investigated the influence of different impact sounds on golfers' perceptions of the balls. From a relationship map generated by Roberts (2002), the following four key influences on 'feel' during putting were determined

- Sharpness of impact sound

- Loudness of impact sound

- Speed of ball from clubface

- Ball hardness

\section{Test Methodology}

The paired comparison technique (Kendall and Babington Smith, 1940) was used to obtain golfers' perceptions of the different balls. Each participant was provided with two ball types at a time and asked to judge the 'feel' of one when compared to the other. For each comparison, five balls of each type, presented as 'Ball Type 1' and 'Ball Type 2' with all distinguishable logos concealed, were provided and the golfer was asked to perform ten $6 \mathrm{~m}$ putts using the standard putter, alternating between ball types. At any point during the comparison and when they were felt confident of their perceptions, they answered the following questions:

- Which impact sound was duller?

- Which ball felt that it left the clubface quicker? 
- Which ball felt harder?

- Which ball had the louder impact sound?

Each golfer performed all ten possible comparisons of the five different ball types. The order in which the comparisons were performed was varied for each golfer. All player tests and procedures were approved by the University Ethics Committee and each player agreed to the conditions set forth in a participant consent form.

Ten golfers, with handicaps ranging from o to 12, participated in the testing. Three were category one golfers (handicaps <5) and seven were category two (handicaps 6-12). The golfers' ages ranged from 22 to 59 years, with a mean age of 36 . All the golfers were righthanded males.

\section{Results}

The responses from each golfer were represented using a pentagon system, where arrows denote the comparison between the two ball types and the direction of the arrow is dependent on the golfer's response to that question (Kendall and Babington Smith, 1940). Figure 11 shows an example of a typical pentagon for perceived sharpness of impact sound. For this participant, ball $\mathrm{C}$ was perceived to have a sharper sound than any of the other balls as all the arrows point towards $C$. For each of the four questions a pentagon was produced for each golfer.

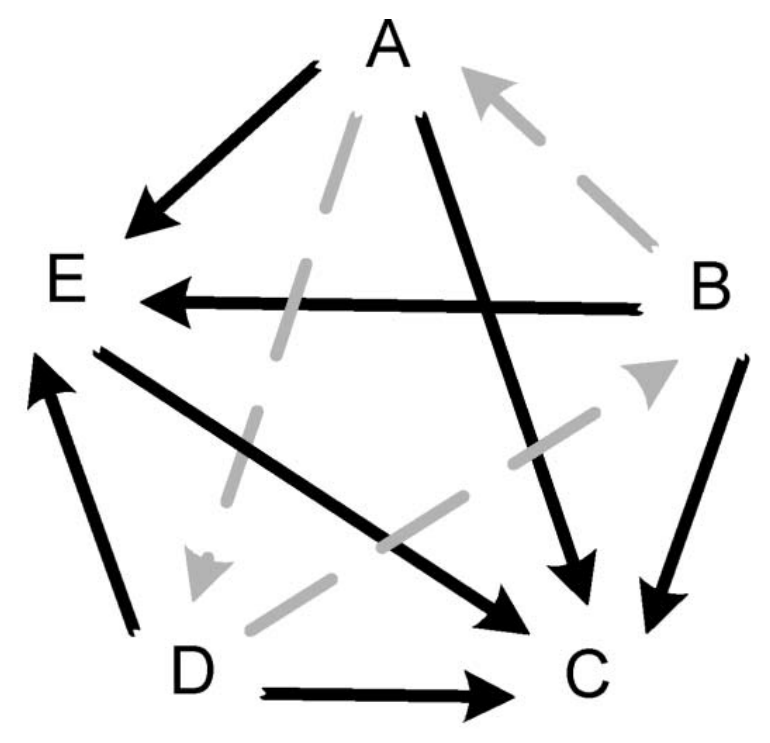

\section{SHARPNESS OF SOUND (arrows point to a duller sound)}

Figure 11. Example of the pentagon system for representing golfer's responses.
The subjective responses for any combination of three objects forms a 'triad' and analysis of each triad in the pentagon allows inconsistent responses to be identified. In Figure 11, for example, the triad ABD is considered to be inconsistent and is referred to as a circular triad (David 1988). This is because, if $D$ was perceived to have a duller sound than $\mathrm{A}$, and $\mathrm{B}$ was perceived to sound duller than $\mathrm{D}$, it then follows that $\mathrm{B}$ should also have been rated as having a duller sound than A.

The Kendall coefficient of consistence, $\zeta$, is based on the ratio of the number of circular triads, $d$, to the number of possible circular triads and can be used as an overall measure of the consistency of a participant's responses (Kendall and Babington Smith, 1940). When the total number of objects, $n$, is odd, the coefficient of consistence is given by Equation (2).

$$
\zeta=1-\frac{24 d}{n^{3}-n}
$$

A value of 1 indicates there are no inconsistencies and as $\zeta$ decreases to zero the inconsistency, as measured by the number of circular triads, increases (David, 1988). A coefficient of consistence greater than 0.75 is generally considered acceptable (Otto et al,. 1999).

The mean coefficients of consistence were $0.91,0.8$, 0.85 and 0.68 for responses to sharpness of sound, speed from the clubface, ball hardness and impact loudness respectively. This implies that the question on perceived loudness of impact sound was answered much less consistently than the other questions. Kendall and Babington Smith (1940) suggested that, 'if an individual observer produces a configuration of preferences, which show inconsistence, there are usually several explanations; he may be an incompetent judge, the objects may be so alike that consistent differentiation is not possible, or his attention may wander during the course of the experiment.' It can be seen in Figure 8 that for a given impact location, there is only 3-4 $\mathrm{dB}(\mathrm{A})$ difference in sound pressure level between the balls. This difference is only just perceivable to humans when the sounds are continuous and loudness discrimination becomes even more difficult when the duration of the sounds is less than one second (Hassell \& Zaveri,1979; Zwicker \& Fastl, 1999). It is likely, therefore, that the inconsistent ratings of loudness of sound were due to similar characteristics of the balls in this respect rather than the golfers were incompetent judges.

The degree of agreement among participants in the way they rated the balls for each of the four questions can be measured using the Kendall coefficient of agreement, $u$, (Siegel and Castellan, 1988). In calculating the agreement, a matrix was formed to summarize the number of times each ball was rated as possessing more of a certain characteristic than the other balls. An example matrix can 
be seen in Table 2, which shows the number of times the ball in each column was rated as feeling harder than the ball in each row. For example, nine out of ten golfers perceived ball $\mathrm{B}$ to be harder than ball $\mathrm{A}$.

Table 2. Matrix constructed to analyse players' perceptions of ball hardness.

\begin{tabular}{|c|c|c|c|c|c|c|}
\hline \multirow{2}{*}{} & \multicolumn{7}{|c|}{ Felt harder } \\
\cline { 2 - 7 } & A & B & C & D & E & Sum \\
\hline A & & 9 & 1.5 & 5.5 & 4 & 20 \\
\hline B & 1 & & o & 2 & 1 & 4 \\
\hline C & 8.5 & 10 & & 9 & 6 & 33.5 \\
\hline D & 4.5 & 8 & 1 & & 5 & 21.5 \\
\hline E & 6 & 9 & 4 & 5 & & 24 \\
\hline Sum & 20 & 36 & 6.5 & 21.5 & 16 & \\
\hline $\begin{array}{c}\text { Rank } \\
\text { Sum }\end{array}$ & 60 & 76 & 46.5 & 64.5 & 56 & \\
\hline
\end{tabular}

The Kendall coefficient of agreement uses values from this table to determine the degree of agreement in the ratings of the balls between participants. The coefficient of agreement, $u$, is given by Equation (3).

$$
u=\frac{8\left(\sum a_{i j}^{2}-k \sum a_{i j}\right)}{k(k-1) n(n-1)}+1
$$

In this case, $a_{i j}$ is the number of times that the ball in row $i$ was perceived to be softer than the ball in column $j$ (the summation is taken above or below the diagonal), $k$ is the number of participants and $n$ is the number of balls (Siegel and Castellan, 1988). If complete agreement exists between judges, $u$ is equal to 1 , but decreases to a minimum value of $-1 /(n-1)$, when $n$ is odd, as disagreement between judges increases.

Coefficients of 0.31 and 0.32 were obtained for sharpness of sound and ball hardness respectively, indicating some agreement between the participants. For loudness of sound and the speed of the ball from the clubface, however, these values reduced to 0.14 and 0.11 respectively, indicating little agreement between the participants. These Kendall coefficients are all significant at the 0.05 level.

The values in Table 2 can also be used to calculate rank sums, which can be used to indicate the relative position of each ball on a continuous scale for each characteristic. The rank sum is calculated by adding the sum of the row frequencies to twice the sum of the column frequencies for each ball. These values are plotted in Figure 12.

The difference between two rank sums that is required for the objects to be considered significantly different is calculated using Tukey's honestly significant difference $(H S D)$, given in Equation (4) (Meilgaard et al. 1999).

$$
H S D=q_{\alpha, n, \infty} \sqrt{\frac{n k}{4}}
$$

Values of $\mathrm{q}$ are tabulated in Meilgaard et al. (1999); for $\mathrm{n}=5$ and a level of significance, $\alpha=0.05,=3.86$, and subsequently a HSD value of 14.9 is obtained.

The significant differences can be summarized as follows. Ball $C$ was perceived to have a duller sound than balls B, D and E. It was also perceived to be softer and leave the putter face slower than balls B and D. Ball B was perceived both to be the hardest ball, and to produce the loudest impact sound, significantly more so than balls A, $\mathrm{C}$ and $\mathrm{E}$. The four feel characteristics also appear to be related. Typically ball $C$ is at one extreme of the scales, whilst ball $\mathrm{D}$ and then ball $\mathrm{B}$ are at the other end. Generally, a ball that was perceived to have a louder, sharper sound was also rated as feeling harder and coming off the clubface quicker and vice versa.

\section{Correlation of subjective responses with measured sound characteristics}

Sharpness of Impact Sound

Ball $C$ was perceived to be the dullest sounding ball, significantly duller than balls B, D and E in particular. Ball $\mathrm{C}$ also had the lowest calculated sharpness, significantly lower than ball B suggesting some correlation between perceived and calculated sharpness. Ball D also had a significantly lower sharpness value than ball B, but this was not perceived by the golfers. There is less correlation with the frequency spectra from the impact tests. Ball B did have the highest frequency peak in the $2-4 \mathrm{kHz}$ range but the order of spectral peaks for balls C, D and E does not correlate well with perceived sharpness.

\section{Ball Speed from Clubface}

Each golfer was consistent in their responses to this question but the agreement between golfers was poor, which suggests that the players were not guessing but that they each used a different basis for their responses. As a result of this lack of agreement, the range of rank sums for the five balls is smaller than for the other questions, so few significant differences emerged between balls.

\section{Ball Hardness}

There was little correlation between perceived ball hardness and the compression values of the balls. Ball $\mathrm{B}$ was perceived to be significantly harder than balls $\mathrm{A}, \mathrm{C}$ and $\mathrm{E}$ and has both the highest compression value and the highest frequency peak in the $2-4 \mathrm{kHz}$ frequency range in the impact tests. Ball $\mathrm{D}$, however, was also rated 


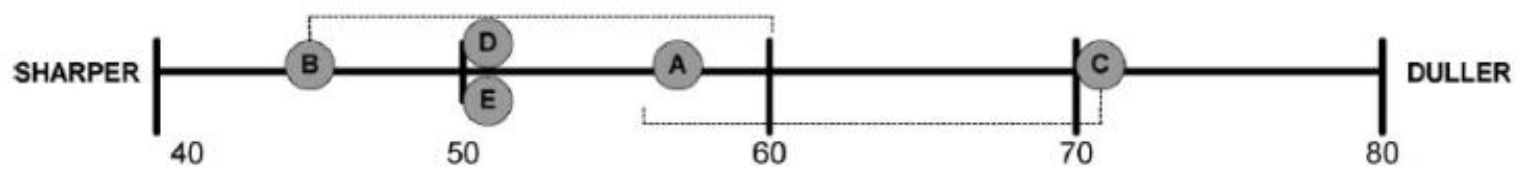

Sharpness of Sound

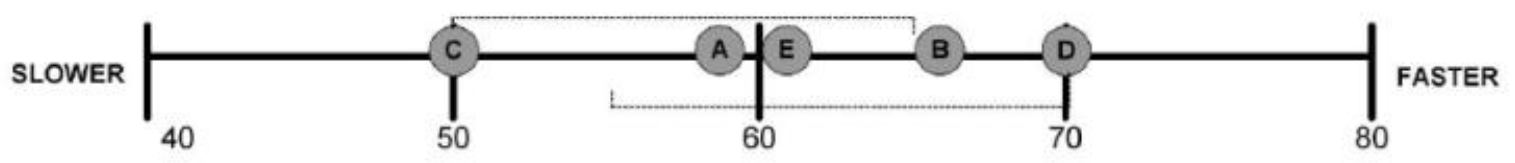

Speed from Clubface

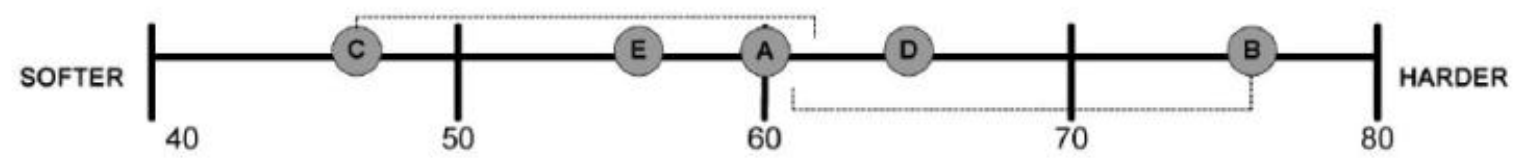

Ball Hardness

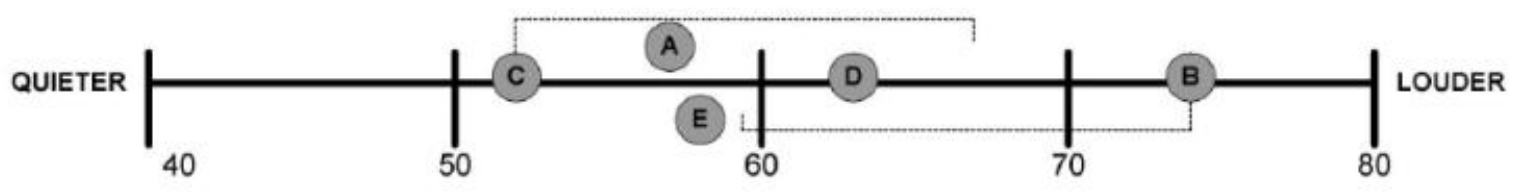

Loudness of Sound

Figure 12. Use of rank sums to locate the different ball types on a scale for each characteristic. Dotted lines denote one HSD.

as one of the hardest feeling balls but had the lowest compression value and the lowest frequency peak in the 2-4 $\mathrm{kHz}$ range. Perceived ball hardness does correlate better with the subjective sound quality metrics of loudness and sharpness. Balls $\mathrm{B}$ and $\mathrm{C}$ were perceived to be the hardest and softest feeling balls, respectively, and also had the highest and lowest values of both loudness and sharpness respectively.

\section{Loudness of Impact Sound}

Ball $B$ was perceived to be significantly louder than balls A, C and E and well separated from ball D (although not significantly); it also produced the highest SPLs and significantly greater loudness values than balls $C$ and $D$. In general, however, the golfers had difficulty identifying loudness differences, resulting in poor coefficients of consistence, probably because there was little difference between the loudness levels of most of the balls.

\section{Conclusions}

This study has examined the sound characteristics of different golf balls and the influence of impact sound on players' perceptions of feel during putting. It is apparent that there is a relationship between the frequency content of an impact sound and the compression value of the ball, which could be used to determine ball properties. In particular, stiffer balls have been found to generate frequency components higher in the range from $2-4 \mathrm{kHz}$ than softer golf balls. The type of putter has also been found to influence the impact sound. In the $0-2 \mathrm{kHz}$ range, little difference could be seen between different ball types but the insert putter generated more low frequency sound components than the standard putter.

In terms of golfers' perceptions of the balls, the results showed that ball B, which produced the greatest loudness and sharpness values, along with the highest frequency component, was also perceived to have the sharpest and loudest impact sound and was rated as feeling the hardest and one of the fastest balls from the clubface. This 
relationship did not hold for all balls, however, as ball $\mathrm{D}$ was perceived to have a similar feel to ball B but its measured sound characteristics were significantly different; they were, in fact, similar to ball $C$ which was rated as having the dullest, quietest sound and felt softest and slowest off the face.

This research suggests that simple metrics such as ball compression are not adequate to quantify how hard a golf ball will feel when struck. Relationships between impact sound characteristics and the feel of a shot have been identified although these relationships are complex and need further investigation. Elite golfers have been found to dislike harder feeling balls for short game play and this paper presents techniques that manufacturers can use to evaluate sound characteristics, along with players' perceptions, of their golf balls as part of the design cycle. As a result, future golf balls can be developed that meet players' requirements for both desirable feel and performance.

\section{Acknowledgment}

The authors would like to thank the golfers who gave up their time to participate in the study. Without their assistance, the study would not have been possible.

\section{References}

Axe J. D., Brown K., Shannon K. (2002) The Vibrational Mode Structure of a Golf Ball. In: Science and Golf IV: World Scientific Congress of Golf (Edited by E. Thain). St Andrews, Scotland. 359367. Routledge Press

David H. A. (1988). The Method of Paired Comparisons: Second Edition. London: Oxford University Press.

Hassall J. R., Zaveri K. (1979). Acoustic Noise Measurements. Naerum: Bruel \& Kjaer.

Hocknell A., Jones R., Rothberg S. J. (1996) Engineering 'feel' in the design of golf clubs. In: The Engineering of Sport: Proceedings of the ist International Conference on the Engineering of Sport (Edited by S. J. Haake). Rotterdam. 333 337. A.A.Balkema

ISO 532. (1975). Acoustics - Method for calculating loudness level. International Organisation for Standardisation.

Kendall M. G., Babington Smith B. (1940). On the Method of Paired Comparisons. Biometrica 41: 324-345.

Meilgaard D. S., Civille B. S., Carr M. S. (1999). Sensory Evaluation Techniques 2nd Edition. London: CRC Press.

Otto N., Amman S., Eaton C., Lake S.(1999) Guidelines fory jury evaluations of automotive sounds. In: Proceedings of the SAE Noise and Vibration Conference and Exposition 1315-1334.

Pelz D. (1989). Putt Like The Pros. New York: Harper Row.

Roberts J. R. (2002). Mechanical \& Psychological influences on the 'feel' of a golf shot [Ph.D thesis]: Loughborough University.

Roberts J.R., Jones, R., Mansfield, N.J. and Rothberg, S.J. (In press). Evaluation of impact sound on the 'feel' of a golf shot. Journal of Sound and Vibration.

Shannon K., Axe J. D. (2002). On the Acoustic Signature of Golf Ball Impact. In: Science and Golf IV: World Scientific Congress of Golf (Edited by E. Thain). St Andrews, Scotland. 368-373. Routledge Press

Siegel S., Castellan N. J. (1988). Nonparametric statistics for the behavioural sciences 2nd Edition. New York: McGraw-Hill.

Zwicker E., Fastl H. (1999). Psychoacoustics: Facts \& Models; Second Edition. Berlin: Springer-Verlag 